\title{
Punto de vista y estructura discursiva argumental en adultos mayores
}

\author{
Cristián Juan Noemi Padilla \\ Profesor Titular de Universidad \\ Departamento de Artes y Letras \\ Universidad de La Serena \\ Benavente 980, La Serena (Chile) \\ E-mail: cnoemi@userena.cl
}

\section{PUNTO DE VISTA Y ES- TRUCTURA DISCURSIVA ARGUMENTAL EN ADUL- TOS MAYORES}

RESUMEN: El trabajo sostiene que la elección del punto de vista que adopta un hablante en un acto de habla argumentativo se encuentra determinada psicosocialmente, situación que afecta igualmente la conformación argumental de sus productos discursivos. A fin de especificar parte de las características psicosociales de adultos mayores de la muestra, se empleó el test Hample (Pizarro y Rodriguez, 2013). Con el propósito de obtener una muestra significativa de textos que permitieran establecer la estructura argumental recurrente de este tipo hablantes, se utilizó el Dilema III del Cuestionario D.I.T. (Rest, 2013). El cruce de información entre ambos instrumentos permitió establecer relaciones significativas entre marcos axiológicos, punto de vista y estructura semántico discursiva. De este modo se observa que los adultos mayores optan mayoritariamente por el punto de vista en contra, por medio de esquema sintomático.

PALABRAS ClAVes: punto de vista; estructura argumental; adultos mayores; características psicosociales; marcos axiológicos.

SUMARIO: 1. Introducción. 2. 2. Marco teórico. 2.1. Punto de vista. 2.2. Esquema psicosocial. 2.3. Estructura. 2.4. Tipo. 3. Metodologia. 4. Análisis. 5. Hallazgos. 6. Conclusiones.

Fecha de Recepción Fecha de Revisión Fecha de Aceptación Fecha de Publicación

\section{POINT OF VIEW AND AR- GUMENTATIVE DISCUR- SIVE STRUCTURE IN OLDER ADULTS}

ABSTRACT: The work argues that the choice of the point of view adopted by a speaker in an act of argumentative speech is psychosocially determined, a situation that also affects the discursive argument configuration. In order to specify part of the psychosocial characteristics of older adults in the sample, the Hample test was used (Pizarro and Rodriguez, 2013). In order to obtain a significant sample of texts that would allow establishing the recurrent argumentative structure in this type of speakers, Dilemma III of the D.I.T. Questionnaire was used (Rest, 2013). Cross-referencing of information between both instruments allowed establishing significant relationships between axiological frameworks, point of view and discursive semantic structure. In this way it is observed that older adults opt mostly for the opposite point of view, by means of a symptomatic scheme.

KEY WORDS: point of view; argumentative structure; older adults; psychosocial characteristics; axiological frameworks.

SUMMARY: 1 . Introduction. 2 . 2. Theoretical framework. 2.1. Point of view. 2.2. Psychosocial scheme. 2.3. Structure. 2.4. Kind. 3. Methodology. 4. Analysis. 5. Findings. 6. Conclusions.

\section{POINT DE VUE ET STRUCTURE DISCURSIVE ARGUMENTATIVE CHEZ LES PERSONNES AGEES}

RÉSUMÉ: Le travail fait valoir que le choix du point de vue adopté par un locuteur dans un acte de discours argumentatif est déterminé par des facteurs psychosociaux, une situation qui affecte également la conformation argumentative de ses produits discursifs. Dans le but de préciser une partie des caractéristiques psychosociales des personnes âgées dans l'échantillon, le test Hample a été utilisé (Pizarro et Rodríguez, 2013). Afin d'obtenir un échantillon significatif de textes permettant d'établir la structure d'argumentation récurrente de ce type de locuteurs, Dilemme III du Questionnaire D.I.T. a été utilisé (Rest, 2013). Le croisement des informations entre les deux instruments a permis d'établir des relations significatives entre les cadres axiologiques, le point de vue et la structure sémantique discursive. De cette façon, on observe que les personnes âgées optent principalement pour le point de vue opposé, au moyen d'un schéma symptomatique.

MOTS CLÉS: point de vue; structure argumenté; adultes majeurs; caractéristiques psychosociales; cadres axiologiques.

SOMMAIRE: 1 . Introduction. 2. 2. Cadre théorique. 2.1. Point de vue. 2.2. Schéma psychosocial. 2.3. Structure. 2.4. Type. 3. Méthodologie. 4. Analyse. 5. Constatations. 6. Conclusions. 


\section{Punto de vista y estructura discursiva argumental en adultos mayores 1}

CRISTIÁn JUAn NoEmi PAdilla

\section{INTRODUCCIÓN}

Aparentemente, la primera inferencia psicosocial que se expresa discursivamente en un acto de habla argumentativo (Marraud, 2018) se formaliza en la macroestructura textual en la forma del llamado punto de vista (Noemi, 2013). Según se sugiere, el punto de vista que adopta un sujeto durante un acto de habla argumentativo se encuentra determinado por variables psicosociales que orientan esta conducta y condicionan al menos parcialmente la estructura discursiva semántica.

La noción de punto de vista puede rastrearse desde el corpus doctrinario de la teoría retórica clásica, por ejemplo, a través del tratamiento del concepto de status (Quintiliano, 1916), hasta trabajos contemporáneos de motivación principalmente pragmadialéctica (van Eemeren y Grootendorst, 2004). Cualquiera sea el caso, ya se conciba el punto de vista como una tensión entre la tesis de la acusación y la de la defensa, o bien como un espacio de desacuerdo entre quienes se involucran en la resolución de un conflicto, las posturas coinciden en que es la disputa la que genera el acto de habla argumentativo y el consecuente despliegue discursivo de las partes involucradas.

Si bien la teoría de la argumentación ha tenido en este sentido un avance conceptual considerable, existe todavía un gran vacio en lo que respecta al conocimiento de la ontogénesis de esta capacidad discursiva (Santibáñez, 2016), particularmente en lo que guarda relación con las variables psicosociales involucradas en la conducta argumentativa del grupo etario considerado.

En este tenor, el trabajo ha pretendido determinar algunas de las principales variables psicosociales asociadas el establecimiento del punto de vista en hablantes adultos mayores chilenos al momento de generar un acto de habla argumentativo, a la vez que describir parte de las características semántico-formales de los constructos discursivos textualizados a partir de tal elección.

Para este propósito, en la sección Marco Teórico se refieren y discuten las nociones de punto de vista, esquema psicosocial y estructura. En la sección Metodología, se da cuenta del tipo de diseño del trabajo y se caracteriza la muestra del corpus. En el apartado Análisis se explica el procedimiento seguido en la aplicación del test Hample (Pizarro y Rodríguez, 2013) y del ins-

\footnotetext{
${ }^{1}$ Este trabajo se enmarca en los proyectos de investigación FONDECYT N ${ }^{\circ} 1170492$ y DIDULS $\mathrm{N}^{\circ}$ PR16211.
} 
trumento Dilema III del Cuestionario D.I.T. (Rest, 2013). En la sección Hallazgos se presentan y comentan las principales tendencias que se observan luego del análisis del corpus, y finalmente en el apartado Conclusión se discuten los principales alcances y limitaciones del trabajo.

\section{MARCO TEÓRICO}

\subsection{PunTo DE VISTA}

Según se esbozó, mutatis mutandis, la noción de punto de vista fue abordada en el marco de la teoría retórica clásica en el contexto de la noción de status, entendida como una propiedad formal de índole semántica común de los discursos de litigio: "/.../ id quod est commune omnibus, qui sit status /.../" (“/.../ aquello que es común para todos; esto es, el status/.../") (Quintiliano, 1916: 326).

El concepto de status representa, en este sentido, una suerte de marca textual de la teoría del caso (Baytelman y Duce, 2004), y en consecuencia un concepto que coincide fuertemente con la noción de punto de vista; esto es, por una parte, la postura a partir del cual el litigante organiza la estructura argumental de su discurso y, por otra, el contenido que asoma cuando los usuarios del lenguaje están en desacuerdo frente a un hecho y expresan su opinión cara a resolver el desacuerdo (van Eemeren \& Grootendorst, 1982).

Ante una situación controversial, en efecto, los hablantes ya sea de manera consciente o subconsciente toman partido por una posición determinada y, si está en su ánimo el resolverla, expresan su opinión a través de un punto de vista que se manifiesta formalmente en una proposición que, en dependencia de la competencia argumentativa disponible (Hammer y Noemi, 2015), se fundamenta por medio de una estructura discursiva de mayor o menor complejidad (Noemi, 2013).

La noción de punto de vista, en este sentido, permite tanto situar psicosocialmente al sujeto que lo emite, como proporcionar conocimiento de aspectos de la estructura psicosocial del contexto, por ejemplo, a través del análisis del espacio de desacuerdo (Jackson, 1992). La noción igualmente permite observar la organización del discurso argumentativo a partir del contexto de desacuerdo, toda vez que el hablante que manifiesta un punto de vista determinado usualmente lo sostiene argumentativamente, proporcionando información sustantiva que lo avala, hecho que afecta la estructura discursiva formal (Plantin, 1996).

\subsection{ESQUEMA PSICOSOCIAL}

Contemporáneamente, la noción de esquema de naturaleza psicosocial ha sido tratada desde dos perspectivas; ya sea, desde una óptica de tipo cognitivo, o bien a partir de un enfoque de naturaleza pragmático. 
Hastings (1963), por ejemplo, desde la primera óptica describe nueve modos de razonamiento que agrupa en tres clases: procedimientos verbales y semánticos; conexiones causales; y argumentos soportados ya sea en conclusiones verbales o causales (Hastings, 1963). En un sentido similar, Perelman y Olbrechts-Tyteca (1989) conciben la noción de topoi, ya como premisas o bien como depósito de argumentos que agrupan en categorías tales como lugares de la cantidad, de la cualidad, del orden, de lo existente, de la esencia, o de la persona. Kienpointner (1987), en esa misma linea, propone una clasificación de esquemas de argumentos en relación con reglas de generalización, a partir de opiniones socialmente aceptadas.

Desde una perspectiva pragmática, Walton et al. (2008), por ejemplo, proponen una categorización de esquemas en contextos conversacionales especificos, a partir del establecimiento de posibles falacias, las que en su opinión no se determinan simplemente de la información vehiculada proposicionalmente, sino que por la situación contextual y dialogal específica, tal cual es el caso, por ejemplo, del argumento de autoridad (Walton, 1997).

En el marco de este trabajo, se asume que un esquema constituye parte de la configuración psicosocial de un sujeto, la que contribuye a pautar sus preferencias estructurales discursivas. Con atención a que un esquema argumentativo refiere pautas estereotipadas de razonamiento (Marraud, 2013; Godden y Walton, 2007) el trabajo ha procurado describir la utilización de esquemas sintomático, analógico y/o instrumental en una muestra de adultos mayores chilenos y su relación con preferencias textuales.

El esquema sintomático representa un modo de razonamiento a partir del cual se concibe a los argumentos como indicios o sintomas que conducen a una determinada conclusión y/o soporte de la tesis (Zeleznikow y Hunter, 1995).

El esquema de razonamiento analógico está basado en el supuesto con arreglo al cual la aceptabilidad de las premisas se transfiere a la conclusión haciendo que se asuma que existe una relación de analogia entre lo que es afirmado en el argumento y lo que es afirmado en el punto de vista (van Eemeren, 2012).

El esquema de razonamiento instrumental concibe los argumentos como medios o instrumentos causales que explican y justifican la tesis o conclusión (Walton et al., 2008); en este sentido, los argumentos son un medio para alcanzar la conclusión, o bien, constituyen una causa que la provoca (van Eemeren, 2014).

\subsection{ESTRUCTURA}

Puesto que la tradición filosófico-lingüística contemporánea se ha orientado más bien sobre los productos que sobre los procesos (Marraud, 2018), la pretensión por referir la estructura del argumento se ha abordado preferentemente desde la perspectiva de la lógica informal o desde una óptica de naturaleza pragmática. 
En el marco de la lógica informal, la naturaleza dialéctica del fenómeno favoreció desde esta perspectiva la generación de varios métodos de descripción del argumento que tuvieron en común una forma de representación arbórea que cuajó en la clásica propuesta de Toulmin (Toulmin,1958), y se extendió hasta modelos formales matemáticos para identificar la estructura del argumento y medir la aceptabilidad de la conclusión Selinger (2014).

Desde una óptica pragmática, los intentos por referir la estructura del argumento han estado asociados, en el marco de la denominada discusión crítica, preferentemente a la llamada teoria pragmadialéctica (van Eemeren y Grootendorst, 2004).

En atención a la tarea discursiva demandada por la metodología que conformó el corpus de este trabajo, se ha concebido la noción de estructura en términos del número de argumentos de una secuencia y el conjunto de sus relaciones (van Eemeren y Grootendorst, 2002). En este sentido, se ha admitido que una argumentación única: “/.../contiene sólo un argumento, generalmente formado por una premisa explícita y una implicita." (van Eemeren y Grootendorst, 2002: 95), y que una argumentación múltiple: “/../puede presentar una combinación de argumentaciones únicas." (van Eemeren y Grootendorst, 2002: 95).

\subsection{TIPO}

Con atención de la perspectiva epistémica que hayan adoptado los distintos enfoques teóricos, la tipología de los argumentos ha recibido un tratamiento análogo al de los esquemas y estructuras.

En el modelo de Perelman y Olbrechts-Tyteca (1989), por ejemplo, se refieren diversos tipos de argumentos categorizados de acuerdo a su naturaleza, en a) argumentos cuasi lógicos en la medida en que se presentan como comparables a razonamientos formales, lógicos o matemáticos; b) y argumentos basados en la estructura de lo real, en el sentido de que se sirven de esta estructura para establecer una solidaridad entre juicios admitidos y otros que se intenta promover.

En el modelo pragmadialéctico, los argumentos son categorizados en relación con la violación de las reglas de discusión (van Eemeren y Grootendorst, 2002). Finalmente, en el contexto de la lógica informal, Walton (1997), por ejemplo, sugiere una categorización a partir de la noción de inferencia que supone el concepto mismo de argumento, en argumentos de tipo abductivos, argumentos de naturaleza causal, argumentos de naturaleza deductiva, y argumentos inductivos.

\section{MetOdOLOGÍA}

El trabajo siguió una metodología mixta cuantitativa y cualitativa, con un tipo de diseño descriptivo. A fin de seleccionar la muestra, ésta se tamizó utilizando los instrumentos Test Minimental y el test de Depresión Geriátrica 
Yesavage, lo que permitió disponer de un grupo de adultos mayores cognitivamente sanos a quienes se les solicitó los respectivos consentimientos informados.

De este modo, se obtuvo una muestra estadísticamente significativa conformada por 243 adultos mayores chilenos de las Regiones de Coquimbo (82), Metropolitana (80) y Bío-Bío (81), a los que se les aplicó el test Hample (Pizarro y Rodríguez, 2013) a fin de identificar sus esquemas psicosociales recurrentes. Para establecer la confiabilidad del instrumento se utilizó el Alfa de Cronbach, que alcanzó un indicador de 0,844. Con la información obtenida, se levantó una base de datos, utilizando el software SPSS, que permitió describir los tipos de esquemas preferentes desde los cuales los adultos mayores generan sus discursos.

Por otra parte, a fin de observar sus puntos de vista y preferencias discursivas (estructura y tipo) se les solicitó a los participantes un ensayo en torno a una situación controversial, para lo cual se utilizó el Dilema III del Cuestionario D.I.T. (Rest, 2013) (ver Anexo). Los discursos fueron luego transcritos y analizados semánticamente utilizando el software Atlas/Ti 7.0.

\section{ANÁlisis}

El instrumento diseñado por Hample (2005) consiste en un cuestionario con preguntas cerradas en combinación con el método de escalamiento Likert, que permite medir la función atribuida a los argumentos de acuerdo a marcos culturales. Si bien el instrumento incluye otras dimensiones tales como edición cognitiva y rasgos de personalidad, este trabajo focalizó la atención en la noción de esquema.

Para completar el análisis, se incluyeron las variables sociométricas de rango etario y género. Para dar cuenta del rango etario, se arbitró la clasificación: Rango1para el tramo 65-70 años, Rango2 para 70-75, Rango3 para 75-80, y Rango4 para más de 80 años. Finalmente, la información cualitativa, procesada en el software SPSS 23, se cruzó con los resultados del análisis textual del Dilema III del cuestionario DIT (Rest, 2013), realizado con ayuda del programa Atlas/Ti 7.0.

\section{HaLlazgos}

5.1. Como puede observarse en la Tabla $N^{\circ} 1$, los adultos mayores de la muestra mayoritariamente adoptaron el punto de vista en contra $(87,7 \%$ vs. $12,3 \%$, respectivamente). Quienes se inclinan por adoptar el punto de vista a favor, lo vehiculan discursivamente, de manera preferente, por medio de esquema Instrumental: $88,8 \%$, como se ejemplifica en (1):

(1) "Sí, por supuesto, porque sigue siendo un delincuente ante la sociedad /..." 
Por otra parte, quienes optan por el punto de vista en contra, emplean mayoritariamente esquema sintomático: $60,6 \%$, como se muestra en (2):

(2) "No, porque el señor López aprendió la lección el mismo y así pudo recuperarse y ayudar a mucha gente. Tendría que empezar nuevamente para ser alguien en la vida".

\begin{tabular}{|c|c|}
\hline Punto de vista & Esquema \\
\hline \multirow{4}{*}{ Favor } & Sintomático \\
$12,3 \%$ & $11,1 \%$ \\
\cline { 2 - 3 } & Instrumental \\
& $88,8 \%$ \\
\cline { 2 - 3 } & Analógico \\
& $0 \%$ \\
\hline \multirow{4}{*}{ Contra } & Sintomático \\
& $60,6 \%$ \\
\cline { 2 - 3 } & Instrumental \\
& $27,2 \%$ \\
\cline { 2 - 3 } & Analógico \\
& $12,1 \%$ \\
& \\
&
\end{tabular}

Tabla 1: Empleo de esquemas a partir de punto de vista

5.2. Los sujetos del rango etario 1 adoptan indistintamente tanto el punto de vista a favor como en contra: $44,4 \%$ y $39,3 \%$, respectivamente. A mayor rango etario, disminuye la tendencia con una inclinación hacia Contra en Rango3, que no obstante se equipara en Rango4 (ver tabla $\mathrm{N}^{\circ} 2$ ).

\begin{tabular}{|c|c|c|c|c|}
\hline Punto de vista & \multicolumn{4}{|c|}{ Rango } \\
\hline & 1 & 2 & 3 & 4 \\
\hline Favor & $44,4 \%$ & $33,3 \%$ & $11,1 \%$ & $11,1 \%$ \\
\hline Contra & $39,3 \%$ & $33,3 \%$ & $21,2 \%$ & $9,0 \%$ \\
\hline
\end{tabular}

Tabla 2: Punto de vista y rango etario

5.3. El género masculino está fuertemente asociado a punto de vista a favor, mientras que el femenino lo está a punto de vista en contra; $66,6 \%$ y $75,7 \%$, respectivamente. La diferencia de punto de vista en género masculino es más tenue comparada con género femenino en el que la tendencia a la oposición es más discreta (ver Tabla $\mathrm{N}^{\circ} 3$ ).

\begin{tabular}{|c|c|c|}
\hline Punto de vista & \multicolumn{2}{|c|}{ Género } \\
\hline & Masculino & Femenino \\
\hline Favor & $66,6 \%$ & $24,3 \%$ \\
\hline Contra & $27,2 \%$ & $75,7 \%$ \\
\hline
\end{tabular}

Tabla 3: Punto de vista y género 
5.4. El tipo de estructura no parece comportar algún vínculo significativo con la postura a favor o en contra representada en el punto de vista. Se observa, sin embargo, preferencia de los adultos mayores por verbalizar su postura a través de Estructura múltiple coordinada y ausencia -con independencia de la postura adoptada- de Estructura múltiple subordinada (ver Tabla $\mathrm{N}^{\circ} 4$ ).

\begin{tabular}{|c|c|c|c|}
\hline Punto de vista & Estructura única & $\begin{array}{c}\text { Estructura múltiple } \\
\text { coordinada }\end{array}$ & $\begin{array}{c}\text { Estructura múltiple } \\
\text { subordinada }\end{array}$ \\
\hline Favor & $44,4 \%$ & $55,5 \%$ & $0 \%$ \\
\hline Contra & $39,3 \%$ & $63,6 \%$ & $0 \%$ \\
\hline
\end{tabular}

Tabla 4: Punto de vista y estructura

5.5. Los tipos de argumentos de los sujetos de la muestra son similares con independencia del punto de vista adoptado (ver Tabla $\mathrm{N}^{\circ} 5$ ). No obstante, el argumento causal está fuertemente vinculado a punto de vista a favor $(68,7 \%)$, según se puede observar en (3) y (4):

(3) "Sí por supuesto, porque sigue siendo un delincuente ante la sociedad".

(4) "Honestamente creo que debe denunciarlo, porque tiene un proceso que cumplir el Sr. González. En este caso ayuda solamente al Sr. López y este debe pagar con la sociedad /.../".

Tanto el argumento ad misericodiam, como se ejemplifica en (5) y (6):

(5) "No porque el Sr. López aprendió la lección él mismo y así pudo recuperarse y ayudar a mucha gente /.../".

(6) "/.../ todos tenemos que tener una segunda oportunidad. Yo creo que no deben denunciarlo porque está arrepentido por lo que hizo”.

como el argumento de reciprocidad -según se observa en (7) y (8)- son más bien característicos del punto de vista en contra (64\%, entre ambos):

(7) "Yo creo que no, porque él supo aprovechar su segunda oportunidad en la vida. Cambió y supo ser honesto y bueno con las personas".

(8) "No porque a todos hay que darles una segunda oportunidad. Creo que este señor cuando hizo su delito era inmaduro y ahora ha cambiado; por lo tanto, el otro señor debiese olvidar lo ocurrido".

\begin{tabular}{|c|c|}
\hline Punto de vista & Tipo de argumento \\
\hline \multirow{2}{*}{ Favor } & Identidad: $6,2 \%$ \\
& Reciprocidad: $12,5 \%$ y de \\
& Causal: $68,7 \%$ \\
& Ad misericordiam: $12,5 \%$ \\
\hline Contra & Identidad: $6,0 \%$ \\
& Reciprocidad: $24,0 \%$ \\
\hline
\end{tabular}




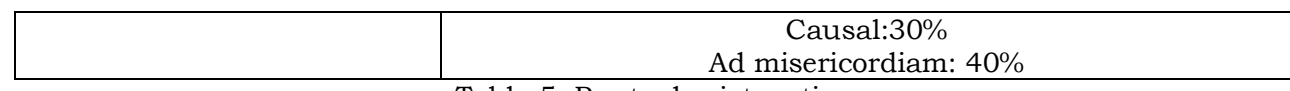

Tabla 5: Punto de vista y tipo

\section{Conclusiones}

Según se propuso, la elección del punto de vista que adopta un hablante en un acto de habla argumentativo se encuentra pautado psicosocialmente, hecho que igualmente motiva preferencias discursivas especificas.

Enfrentados al dilema moral en cuestión (ver Anexo) y a la necesidad avalar argumentativamente su elección, los adultos mayores de la muestra optan mayoritariamente, por el punto de vista en contra $(87,7 \%)$, empleando discursivamente para ello preferentemente un esquema psicosocial de tipo sintomático $(60,6 \%)$.

En términos generales, los sujetos de la muestra, a partir del valor que le atribuyen a una determinada circunstancia moral (Frondizi, 1972), y debido probablemente a condicionantes de socialización contextuales específicas, se sitúan preferentemente en un marco axiológico de naturaleza cardinal y/o teologal (Aristóteles, 2006), por lo que avalan discursivamente su punto de vista por medio de argumento ad misericodiam y argumento de reciprocidad (64\%, entre ambos).

Esto pareciera explicar la diferencia de género en relación con el punto de vista adoptado. Según se observó, el género masculino está fuertemente asociado a punto de vista a favor $(66,6 \%)$, mientras que el género femenino lo está hacia punto de vista en contra $(75,7 \%)$. Al parecer, ambos géneros, en atención a factores de socialización disímiles y a funciones sociales diferenciadas se ubican en consecuencia en marcos axiológicos distintos, lo que tiene consecuencias en el esquema y la organización discursiva consecuente que fundamenta su postura frente a una situación controversial.

En este sentido, el género masculino se asocia a un marco axiológico pragmático (Aristóteles, 2006), por lo que fundamenta la elección de su punto de vista, preferentemente a partir de un esquema instrumental que se expresa en la macroestructura discursiva mayoritariamente en la forma de argumento causal (68,7\%). El género femenino, por el contrario, se asocia a un marco axiológico cardinal (Aristóteles, 2006), que pauta la elección de un punto de vista a partir de esquema sintomático que se manifiesta discursivamente en términos de argumento ad misericordiam y de reciprocidad.

Como se indicó, es posible advertir preferencia de los adultos mayores por verbalizar su postura a través de estructura única y de estructura múltiple coordinada, sin comprobarse formalización de estructura múltiple subordinada. La comparación de esta muestra con otros grupos etarios evaluados precedentemente (Noemi y Rossel, 2017), pareciera advertir sobre baja competencia discursiva por parte de los adultos mayores encuestados para sostener un punto de vista, a la hora de afrontar argumentativamente una situación controversial. 
Con atención al hecho de que, por otra parte, ha sido igualmente posible notar correlación entre capacidades inferenciales y complejidad textual en muestras de jóvenes universitarios (Hammer y Noemi, 2015), el comportamiento discursivo de esta muestra de adultos mayores alerta respecto de sus capacidades lingüístico-psicosociales, cara a las demandas argumentativas que el actual contexto social les impone.

\section{REFERENCIAS}

ARISTÓTELES (2006): Ética a Nicómaco, Buenos Aires: Gradifco.

BAYTELMAN, A. \& DUCE, M. (2004): Manual de litigación en juicios orales, Santiago de Chile: Centro de Estudios de Justicia de Las Américas.

FRONDIZI, R. (1972): ¿Qué son los valores?: una introducción a la axiología, México: Fondo de Cultura Económica.

GODDEN, D., \& WALTON, D. (2007): "Advances in the Theory of Argumentation Schemes and Critical Questions", Informal Logic, 27, pp. 267-292.

HAMMER, L. \& NOEMI, C. (2015): "Relación entre pensamiento crítico y complejidad discursiva en estudiantes universitarios", Onomázein, 32, pp.184-197.

HAMPLE, D. (2005): Arguing: Exchanging Reasons Face to Face, Mahwah, NJ: Erlbaum.

HASTINGS, A. (1963): A Reformulation of the Modes of Reasoning in Argumentation, (Tesis doctoral), Northwestern University, Illinois.

JACKSON, S. (1992): "Virtual standpoints and the pragmatics of conversational argument". En F. van Eemeren, R. Grootendorst, J.A. Blair, \& C.A. Willard (eds.): Argumentation illuminated. Amsterdam: Sic Sat, pp. 260-269.

KIENPOINTNER, M. (1987): "Towards a typology of argumentative schemes”. En F. Van Eemeren, R. Grootendorst, J. A. Blair, \& C. A. Willard (eds.): Argumentation: Across the lines of discipline. Proceedings of the conference on argumentation 1986. Providence: Foris Publications, pp. 275-287.

MARRAUD, H. (2013): ¿Es lógic@? Análisis y evaluación de argumentos, Madrid: Cátedra.

MARRAUD, H. (2018). "Prácticas lingüísticas y prácticas argumentativas". En C. Noemi (ed.): Perspectivas sobre el significado. Desde lo biológico a lo social. Editorial Universidad de La Serena, pp. 85-109.

NOEMI, C. (2013): "Aproximación teórica a la noción de complejidad argumentativa", Logos: revista de lingüistica, filosofía y literatura, 22, 2, pp. 256-271.

NOEMI, C. \& ROSSEL, S. (2017): "Competencia argumentativa psicosocial: esquemas, estructura y tipos de argumentos en estudiantes universitarios chilenos", Revista Lenguaje, Vol. 45, $\mathrm{N}^{\circ}$ 1, pp. 1-23.

PERELMAN, CH. \& OLBRECHTSTYTECA, L. (1989): Tratado de la argumentación: la nueva retórica, Madrid: Gredos.

PIZARRO M. \& RODRÍGUEZ F. (2013): Medición cuantitativa de teoría de la argumentación. (Tesis de grado para optar al título de sociólogo). Instituto de Sociología, Pontificia Universidad Católica de Chile. 
PLANTIN, $\quad$ CH. (1996): L'argumentation, Paris: Le Seuil.

QUINTILIANO, M. (1916): Instituciones oratorias, Madrid: Librería de Perlado y Páez.

REST, W. (2013): Cuestionario de Problemas Socio- morales D.I.T. Madrid: Darwf.

SANTIBÁÑEZ, C. (2016): "Mindreading, representación, inferencia y argumentación". En Cristián Santibáñez (Compilador): Ecología argumentativa universitaria: desde la realidad a los conceptos. Concepción: Cosmigonon, pp. 1952.

SELINGER, M. (2014): “Towards Formal Representation and Evaluation of Arguments", Argumentation, 28, pp. 379393

TOULMIN, S. (1958): The uses of argument. Cambridge: Cambridge University Press.

VAN EEMEREN, F. (2012): "The Role of Logic in Analyzing and Evaluating Argumentation". En J. Ribeiro (ed.): Inside arguments: logic and the study of argumentation. Cambridge: Cambridge Scholars Publishing, pp. 139155.
VAN EEMEREN, F. (2014): La argumentación en la vida politica y juridica, Universidad de Chile: LOM.

VAN EEMEREN F. \& GROOTENDORST, R. (1982): “The speech acts of arguing and convincing in externalized discussions", Journal of pragmatics, 6, pp. 1-24.

VAN EEMEREN, F. \& GROOTENDORST, R. (2002): Argumentación, comunicación y falacias, Ediciones Universidad Católica de Chile.

VAN EEMEREN, F. \& GROOTENDORST, R. (2004): A Systematic Theory of Argumentation. The Pragma-dialectical Approach, Cambridge: Cambridge University Press.

WALTON, D., REED, C. \& MACAGNO, F. (2008): Argumentation schemes, Nueva York: Cambridge University Press.

WALTON, D. (1997): Appeal to Expert Opinion. University Park: The Pennsylvania State University Press.

ZELEZNIKOW, J. \& HUNTER, D. (1995): "Deductive, inductive and analogical reasoning in legal decision support systems", Information \& Communications Technology Law, 4 (2), pp. 141159. 


\begin{abstract}
ANEXo
Dilema III del Cuestionario D.I.T. (Rest, 2013).

\section{- Por favor, lea el siguiente párrafo, y escriba su opinión en relación a la consulta que se hace}

"Un hombre había sido condenado a 10 años de prisión. Después de un año escapó del centro penitenciario cambiándose el nombre por López. Durante ocho años trabajó duramente y, poco a poco, pudo ahorrar el dinero suficiente para montar su propio negocio. Era honesto con sus clientes. Pagaba altos salarios a sus trabajadores y daba la mayor parte de sus beneficios para obras de caridad. Pero un día el señor González, un antiguo vecino de López, le reconoció como el hombre que había escapado de la prisión ocho años antes y al que la policía estaba buscando. ¿Debería el señor González denunciar al señor López a la policía e ir éste de nuevo a prisión?”
\end{abstract}

- ¿Cree usted que debe el Señor González denunciar al señor López? Fundamente su opinión 